\title{
Potencial de rendimento da soja durante a ontogenia em razão da irrigação e arranjo de plantas ${ }^{(1)}$
}

\author{
André Roberto Maehler(2), João Leonardo Fernandes Pires(2), José Antonio Costa ${ }^{(2)}$ e Felipe Gutheil Ferreira ${ }^{(3)}$
}

Resumo - Estimativas do potencial de rendimento (PR) da soja podem ser feitas durante a ontogenia pela quantificação das estruturas reprodutivas, de modo a avaliar o efeito de fatores ambientais e práticas de manejo na produção e fixação dessas estruturas. O objetivo deste trabalho foi determinar o efeito da disponibilidade hídrica e arranjo de plantas no PR e seus componentes, durante a ontogenia de duas cultivares de soja. Os tratamentos constituíram-se de dois regimes hídricos (irrigado e não irrigado), duas cultivares (BRS 137 e BRS 138) e três espaçamentos entre linhas $(20 \mathrm{~cm}, 40 \mathrm{~cm}$ e 20 e $40 \mathrm{~cm}$ em linhas pareadas). O PR médio foi de $15.295 \mathrm{~kg} \mathrm{ha}^{-1}$ no estádio de florescimento; $12.325 \mathrm{~kg} \mathrm{ha}^{-1}$ na formação de legumes; $5.508 \mathrm{~kg} \mathrm{ha}^{-1}$ no início do enchimento de grãos e $4.315 \mathrm{~kg} \mathrm{ha}^{-1} \mathrm{na}$ maturação. $\mathrm{O}$ tratamento irrigado apresentou PR mais elevado em razão da maior produção e fixação de estruturas reprodutivas, mais grãos por legume e grãos mais pesados do que o tratamento não irrigado. Não houve diferença significativa entre espaçamentos. O PR da cultivar BRS 137 é mais elevado do que o da BRS 138, em virtude da menor abscisão de flores, grãos mais pesados e maior número de grãos por legume.

Termos para indexação: Glycine max, etapa de desenvolvimento, disponibilidade hídrica, componentes de rendimento, grão.

\section{Potential yield of soybean during the ontogeny according to water availability and plant arrangement}

\begin{abstract}
The quantification of reproductive structures during the ontogeny allows to estimate soybean yield potential (YP), making possible to quantify the effect of environmental factors and management practices on the production and fixation of these structures. This work aimed to determine the effect of water availability and plant arrangement on the YP and its components, during ontogeny of two soybean cultivars. Treatments were two water availability (irrigated and non irrigated), two cultivars (BRS 137 and BRS 138), and three row spacings (20 cm, $40 \mathrm{~cm}$ and 20 and $40 \mathrm{~cm}$ in skip row). The average YP obtained was of 15,295 kg ha-1 at blooming; $12,325 \mathrm{~kg} \mathrm{ha}^{-1}$ during legume formation; $5,508 \mathrm{~kg} \mathrm{ha}^{-1}$ at the beginning of grain filling and 4,315 $\mathrm{kg} \mathrm{ha}^{-1}$ in maturation phase. The irrigated treatment had higher YP, due to the larger flower and pod production and fixation, more grains per pod and heavier grains, in relation to non irrigated treatment. There was no significant difference among the row spacings tested. The cultivar BRS 137 presented greater YP during the ontogeny than BRS 138, due to less flower abortion, heavier grains and more grains per pod.
\end{abstract}

Index terms: Glycine max, developmental stages, water availability, yield components, grain.

\section{Introdução}

A soja é uma cultura de grande importância socioeconômica para o Rio Grande do Sul e para o

\footnotetext{
(1) Aceito para publicação em 30 de julho de 2002.

(2) Universidade Federal do Rio Grande do Sul (UFRGS), Fac. de Agronomia, Dep. de Plantas de Lavoura, Caixa Postal 776, CEP 90001-970 Porto Alegre, RS. Bolsista do CNPq. E-mail: amaehler@abinet.com.br, piresj1@vortex.ufrgs.br, jamc@ufrgs.br

(3)UFRGS, Fac. de Agronomia, Dep. de Plantas de Lavoura. Bolsista da Fapergs. E-mail: ferreirafg@yahoo.com.br
}

Brasil. Apesar de todo o progresso que a pesquisa tem alcançado com cultivares com maior potencial de rendimento, o desenvolvimento e rendimento da cultura podem ser limitados por estresses de água durante estádios críticos do seu ciclo.

Estudos utilizando irrigação em estádios de desenvolvimento da soja têm sido realizados de forma a determinar quais os períodos críticos à deficiência de água. A maioria dos resultados indica que a sensibilidade da cultura é mínima durante a fase vegetativa, a não ser que a severidade limite muito o porte da planta (Boerma \& Ashley, 1982), e máxima 
durante o período reprodutivo, principalmente na formação de legumes e enchimento de grãos (Korte et al., 1983b). Os efeitos da deficiência hídrica sobre o rendimento vão depender da intensidade, duração, época de ocorrência e da interação com outros fatores determinantes da expressão do rendimento final (Queiroz et al., 1996).

Sionit \& Kramer (1977) verificaram que plantas submetidas à deficiência hídrica durante a formação de flores tiveram o período de florescimento encurtado e produziram menos flores e legumes pelo abortamento de flores. Falta de água durante o período de enchimento reduz o tamanho e o peso dos grãos, por causa da diminuição do suprimento de fotoassimilados pela planta ou da inibição do metabolismo do grão (Sionit \& Kramer, 1977; Salinas et al., 1996). A irrigação pode aumentar o peso dos grãos (Thomas \& Costa, 1994), mas a taxa de enchimento é pouco alterada pela disponibilidade hídrica à planta (Westgate et al., 1989).

A irrigação aumenta o número de legumes por planta, mas o número de grãos por legume é pouco afetado pela deficiência hídrica em cultivares com hábito de crescimento determinado, pois esta característica é intrínseca ao genótipo e pouco influenciada pelo ambiente (Thomas \& Costa, 1994).

Estimativas do potencial de rendimento podem ser feitas durante a ontogenia por meio da quantificação das estruturas reprodutivas (flores e legumes), possibilitando verificar o efeito de fatores ambientais e práticas de manejo sobre a produção e fixação dessas estruturas (Pires et al., 2000). Assim, Ventimiglia et al. (1999) relatam que, se todas as flores presentes em R2 (florescimento) se transformassem em legumes, o rendimento poderia ter sido de $18.000 \mathrm{~kg} \mathrm{ha}^{-1}$, ao passo que se todos os legumes formados até R5 (início do enchimento de grãos) alcançassem a maturação, o rendimento seria de $10.000 \mathrm{~kg} \mathrm{ha}^{-1}$, e o rendimento alcançado em R8 foi de $4.600 \mathrm{~kg} \mathrm{ha}^{-1}$. Este potencial foi influenciado por níveis de fertilidade do solo e pelos espaçamentos entre linhas.

Do total de flores produzidas, $40 \%$ a $80 \%$ são abortadas no florescimento e início da formação de legumes (Hansen \& Shibles, 1978). A queda de legumes ocorre em intensidade muito menor que o aborto de flores (Dominguez \& Hume, 1978), em virtude do custo das fontes carbonadas e nitrogenadas para a formação desses órgãos, que são pequenas comparadas com o necessário para a formação inicial dos legumes (Loomis \& Connor, 1992). Segundo Marchezan \& Costa (1983), para a obtenção de rendimentos elevados seria importante selecionar cultivares com maior capacidade de fixação de legumes.

Entretanto, alto potencial de rendimento na floração, representado pela produção de grande número de flores, não necessariamente identifica uma cultivar eficiente na fixação de estruturas reprodutivas resultando em rendimento de grãos elevado (Navarro Junior, 1998).

O objetivo deste trabalho foi quantificar o potencial de rendimento em quatro estádios reprodutivos de duas cultivares de soja, em razão da disponibilidade hídrica e do arranjo de plantas.

\section{Material e Métodos}

O experimento foi realizado na Estação Experimental Agronômica da Universidade Federal do Rio Grande do Sul (EEA/UFGRS), localizada na região fisiográfica da Depressão Central, Município de Eldorado do Sul, no ano agrícola 1998/99. A precipitação pluvial média anual é de $1.440 \mathrm{~mm}$, apresentando, freqüentemente, problemas de deficiência hídrica nos meses de novembro a março (Bergamaschi \& Guadagnin, 1990).

$\mathrm{O}$ solo da área experimental pertence à unidade de mapeamento São Jerônimo, classificado como Argissolo Vermelho distrófico típico, de acordo com Embrapa (1999), com as seguintes características $(0-20 \mathrm{~cm})$ : argila, $350 \mathrm{~g} \mathrm{~kg}^{-1}$; $\mathrm{pH}, 4,8 ; \mathrm{P}, 13 \mathrm{mg} \mathrm{kg}^{-1}$; $\mathrm{K}, 149 \mathrm{mg} \mathrm{kg}{ }^{-1}$; MO, $20 \mathrm{~g} \mathrm{~kg}^{-1}$; Al, 0,7 cmol $_{\mathrm{c}} \mathrm{L}^{-1}$; CTC, 8,7 cmol $_{\mathrm{c}} \mathrm{L}^{-1}$.

O delineamento experimental utilizado foi o de blocos completamente casualizados, sendo os tratamentos arranjados em parcelas subsubdivididas, com quatro repetições. Os tratamentos constituíram-se em dois regimes hídricos (irrigado e não irrigado), nas parcelas principais; duas cultivares (BRS 137, com ciclo de 129 dias, $78 \mathrm{~cm}$ de altura e $17,1 \mathrm{~g}$ de peso de 100 sementes; e BRS 138, com ciclo de 126 dias, $73 \mathrm{~cm}$ de altura e $14,4 \mathrm{~g}$ de peso de 100 sementes) nas subparcelas e três espaçamentos entre linhas ( $20 \mathrm{~cm}, 40 \mathrm{~cm}$ e 20 e $40 \mathrm{~cm}$ em linhas pareadas), arranjados nas subsubparcelas. As subsubparcelas experimentais tinham $4 \times 6 \mathrm{~m}$, considerando-se como bordadura uma linha em cada extremidade lateral e $0,5 \mathrm{~m}$ em cada cabeceira. 
O experimento foi instalado em semeadura direta, em solo com cobertura de $5 \mathrm{t} \mathrm{ha}^{-1}$ de aveia-preta (Avena strigosa) no início do experimento. A adubação realizada de acordo com Bartz (1994), constou da aplicação a lanço de $150 \mathrm{~kg} \mathrm{ha}^{-1}$ da fórmula 5-20-30.

As sementes foram tratadas e submetidas à inoculação de estirpes específicas de Bradyrhizobium japonicum em meio turfoso. A semeadura foi realizada em novembro, dentro da época recomendada preferencial, com uma semeadora de parcelas, regulada para distribuir as sementes nos espaçamentos desejados. Aos 15 dias após emergência, no estádio V2 (segundo nó, de acordo com Costa \& Marchezan, 1982), realizou-se o desbaste, ajustando-se a população para 40 plantas $\mathrm{m}^{-2}$.

O monitoramento da umidade do solo foi feito com 16 tensiômetros instalados a $20 \mathrm{~cm}$ de profundidade. Efetuouse a irrigação (com aspersores fixos com raio de ação de $3 \mathrm{~m}$ e vazão média de $40 \mathrm{~mm} / \mathrm{h}$ ) na emergência das plantas e sempre que a tensão de água do solo atingia $-0,5$ bar, a partir de 45 dias após a emergência (período vegetativo) até o máximo volume de grãos (R6).

Para estimar o potencial de rendimento realizaram-se determinações pelo mapeamento de plantas (Costa, 1993), em cinco plantas marcadas, em sequiência na linha, de cada subsubparcela, fazendo-se as avaliações nos estádios R2 (florescimento), R4 (formação de legumes), R5 (início do enchimento de grãos) e R8 (maturação), quando cada cultivar atingia os referidos estádios (caracterizados conforme Costa \& Marchezan,1982).

Quantificou-se o número de flores em R2, número de flores e legumes em R4 e número de legumes em R5. Em R8 determinaram-se o número de legumes com zero, um, dois e três grãos e o peso de 100 grãos. Os dados obtidos em R8 foram utilizados para calcular o rendimento de grãos no final do ciclo e o potencial de rendimento em $\mathrm{R} 2$, R4 e R5, quando aplicados aos valores de flores e legumes obtidos nestes estádios. Essa estimativa é a representação do rendimento que seria obtido se as plantas conseguissem manter todas as flores presentes em R2, e todas as estruturas reprodutivas presentes em R4 e R5 se flores e legumes chegassem ao final do ciclo com a proporção de legumes com zero, um, dois e três grãos e o peso de 100 grãos similar ao obtido no estádio R8.

O número de legumes com um, dois e três grãos e sem grãos foi obtido pela contagem desses legumes na amostra de cinco plantas por subsubparcela e depois transformados para $\mathrm{m}^{2}$ e porcentagem. O peso de 100 grãos foi realizado em amostras de 50 grãos, oriundas de legumes com um, dois e três grãos, separadamente.

Os resultados obtidos foram submetidos à análise da variância, pelo teste $\mathrm{F}$ a $5 \%$, e as médias comparadas pelo teste unilateral de Dunnett a 5\% de probabilidade.

\section{Resultados e Discussão}

O ano ocorrido, do ponto de vista meteorológico, pode ser considerado como típico da região, durante o ciclo da soja. A precipitação pluvial média mensal foi de $93 \mathrm{~mm}$, apresentando grandes variações, com ocorrência nos meses de novembro, janeiro e março das menores precipitações. $\mathrm{O}$ balanço hídrico evidenciou deficiência de $330 \mathrm{~mm}$ durante todo o ciclo, sendo mais intensa nos estádios vegetativo, florescimento e enchimento de grãos (Figura 1). No tratamento com suplementação hídrica, a deficiência foi reduzida por irrigações $(240 \mathrm{~mm})$, que supriram parte das necessidades totais de água durante o ciclo.

A deficiência hídrica alterou o ciclo da cultura. Até R5, não houve diferença na duração dos estádios reprodutivos entre os tratamentos irrigado e não irrigado. A partir de R5, o tratamento irrigado levou 12 dias a mais do que o não irrigado para atingir a maturação, resultando em maior período de enchimento de grãos.

Os dados de potencial de rendimento e seus componentes em relação ao fator espaçamento entre linhas não diferiram estatisticamente. Portanto, os resultados que seguem contemplam os três espaçamentos de forma conjunta, analisando os efeitos de regime hídrico e cultivares, sem interação.

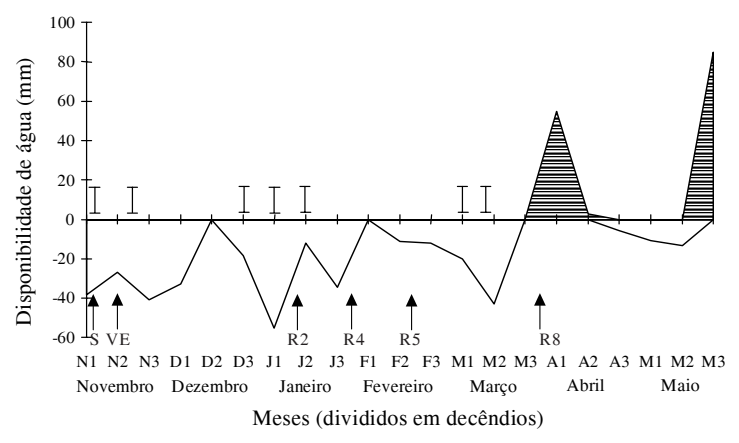

Figura 1. Extrato do balanço hídrico ( $\square$ : deficiência; घ: excesso) segundo Thorntwaite \& Mather (Rolim et al., 1998) para capacidade de armazenamento de água no solo de $50 \mathrm{~mm}$. S: semeadura; VE: emergência; R2: florescimento; R4: formação de legumes; R5: início do enchimento de grãos; R8: maturação; I: irrigação. Eldorado do Sul, RS, 1998/1999. 
Na média dos tratamentos, se todas as flores presentes em R2 tivessem gerado legumes e chegassem à maturação, o potencial de rendimento seria de $15.295 \mathrm{~kg} \mathrm{ha}^{-1}(100 \%)$. Se todas as flores e legumes presentes em R4 e os legumes presentes em R5 tivessem chegado à maturação, o potencial de rendimento seria de $12.325 \mathrm{~kg} \mathrm{ha}^{-1}(80,58 \%)$ e $5.508 \mathrm{~kg} \mathrm{ha}^{-1}(36,01 \%)$, respectivamente. $\mathrm{O}$ potencial de rendimento médio em R8 foi de $4.315 \mathrm{~kg} \mathrm{ha}^{-1}(28,21 \%)$.

Nos estádios R4, R5 e R8, o potencial de rendimento estimado foi maior no tratamento irrigado, evidenciando que a suplementação hídrica reduz a perda do potencial (Figura 2). O padrão de perda do potencial de rendimento foi semelhante nos dois regimes hídricos, apesar de o tratamento irrigado ter partido de um nível mais elevado. Esses resultados indicam que a limitação do suprimento de fotoassimilados, provocada pela deficiência hídrica (Figura 1), pode ter acentuado o aborto de flores e a abscisão de legumes na tentativa de a planta manter equilíbrio entre a produção de fotoassimilados e a fixação de estruturas reprodutivas.

Na maturação, o rendimento foi de $5.189 \mathrm{~kg} \mathrm{ha}^{-1}$, com irrigação, e $3.440 \mathrm{~kg} \mathrm{ha}^{-1}$, sem irrigação.

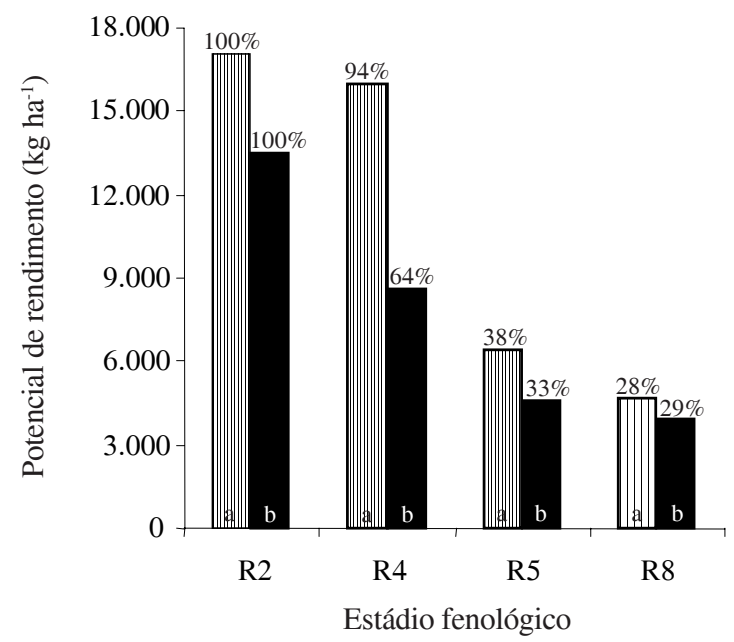

A deficiência hídrica de $85 \mathrm{~mm}$ (Figura 1), que ocorreu entre o segundo decêndio de fevereiro e o segundo decêndio de março, coincidiu com a formação de legumes e enchimento de grãos das plantas, contribuindo para a redução de $34 \%$ no potencial do tratamento não irrigado.

Na comparação entre as cultivares, a BRS 137 apresentou potencial de rendimento mais elevado do que a BRS 138 desde o florescimento até a maturação (Figura 2). Existe grande variabilidade entre cultivares quanto ao número de flores produzidas, legumes produzidos e fixados (Hansen \& Shibles, 1978). Diversos fatores podem interferir no potencial de rendimento, como a deficiência hídrica (Sionit \& Kramer, 1977), limitação da disponibilidade de produtos fotoassimilados (Loomis \& Connor, 1992) e o controle genético (Schaik \& Probst, 1958). Navarro Junior (1998), Ventimiglia et al. (1999), e Pires et al. (2000) verificaram em outras cultivares que tanto fatores ambientais como práticas de manejo influenciam o potencial de rendimento da soja, e que há diferenças entre os genótipos.

A redução do potencial de rendimento entre os estádios R2 e R4 foi de 6\% na BRS 137 e 36\% na

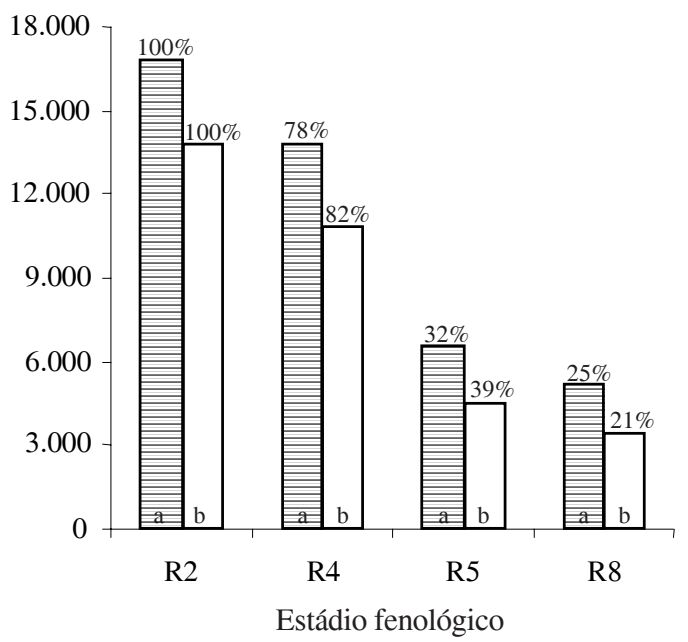

Figura 2. Estimativa do potencial de rendimento de grãos das cultivares de soja BRS 137 ( $\square$ ) e BRS 138 (ם), em dois regimes hídricos ( $\square$ : irrigado; $\square$ : não irrigado) e em porcentual do rendimento máximo. Médias com a mesma letra, em cada estádio fenológico, não diferem entre si pelo teste unilateral de Dunnet a $5 \%$ de probabilidade. R2: florescimento; R4: formação de legumes; R5: início do enchimento de grãos; R8: maturação. Eldorado do Sul, RS, 1998/1999. 
BRS 138. De R4 para R5 e de R5 para R8 ocorreram reduções, respectivamente, de $60 \%$ e $27 \%$ no potencial da BRS 137 e de $47 \%$ e $14 \%$ na BRS 138 . Esse comportamento pode significar vantagem competitiva da cultivar BRS 138, pois ela ajusta seu potencial de rendimento mais cedo, por meio da abscisão de flores e legumes recém-formados, que envolve menor custo energético (Loomis \& Connor, 1992). Porém, essa vantagem não resultou em maior rendimento da cultivar BRS 138.

A produção de flores e legumes em grande quantidade no início do período reprodutivo da cultivar BRS 137 lhe confere elevado potencial, permitindo aproveitar melhor as condições de ambiente e regular mais tarde o potencial de rendimento, apesar de envolver maior demanda de fotoassimilados para manutenção das estruturas reprodutivas por mais tempo.

Os componentes do rendimento foram influenciados pelo regime hídrico e pela cultivar, com interação desses fatores em relação ao número de grãos por legume.

O número de legumes por área é determinado durante os estádios reprodutivos iniciais, e é o componente do rendimento mais importante (Board et al., 1992). O tratamento irrigado apresentou, em média, 256 legumes férteis $\mathrm{m}^{-2}$ a mais do que o tratamento não irrigado (Tabela 1). A irrigação, de modo geral, promove aumento do número de legumes por planta (Thomas \& Costa, 1994). Nesse caso, a irrigação teve grande importância, pois a deficiência hídrica ocorrida em fevereiro coincidiu com os estádios R4

Tabela 1. Legumes com zero, um, dois e três grãos das cultivares de soja BRS 137 e BRS 138, em dois regimes hídricos. Eldorado do Sul, RS, 1998/1999(1).

\begin{tabular}{lcccc}
\hline Legumes $\mathrm{m}^{-2}$ & BRS 137 & BRS 138 & Não irrigado & Irrigado \\
\hline Sem grão & $111 \mathrm{a}$ & $106 \mathrm{a}$ & $122 \mathrm{a}$ & $96 \mathrm{~b}$ \\
Um grão & $171 \mathrm{~b}$ & $271 \mathrm{a}$ & $240 \mathrm{a}$ & $203 \mathrm{~b}$ \\
Dois grãos & $642 \mathrm{a}$ & $681 \mathrm{a}$ & $617 \mathrm{a}$ & $706 \mathrm{a}$ \\
Três grãos & $467 \mathrm{a}$ & $474 \mathrm{a}$ & $368 \mathrm{~b}$ & $572 \mathrm{a}$ \\
\hline${\text { Legumes } \mathrm{m}^{-2}}^{-2}$ & $1.391 \mathrm{a}$ & $1.532 \mathrm{a}$ & $1.347 \mathrm{~b}$ & $1.577 \mathrm{a}$ \\
${\text { Legumes férteis } \mathrm{m}^{-2}}^{1.280 \mathrm{a}}$ & $1.426 \mathrm{a}$ & $1.225 \mathrm{~b}$ & $1.481 \mathrm{a}$ \\
\hline
\end{tabular}

${ }^{(1)}$ Em relação ao regime hídrico e a cultivar, médias seguidas da mesma letra, na linha, não diferem entre si pelo teste unilateral de Dunnett a 5\% de probabilidade; os dados de cultivar representam as médias dos dois regimes hídricos e dos três espaçamentos entre linhas, e os dados de regime hídrico representam as médias das duas cultivares e de três espaçamentos entre linhas. e R5, momento em que o número de legumes estava sendo definido (Figura 1). O aumento do número de legumes vazios e com um grão, em detrimento de legumes contendo três grãos, ocorreu em virtude do menor suprimento de água e assimilados durante o período de formação de legumes e enchimento de grãos.

Em relação às cultivares, não houve diferença significativa quanto ao número total de legumes $\mathrm{m}^{-2}$ (Tabela 1), o que evidencia que as duas cultivares têm padrão semelhante quanto ao número de legumes por área, e a proporção destes, contendo dois e três grãos ou sem grãos, na média das duas condições de disponibilidade hídrica.

A interação entre cultivar e regime hídrico indica não haver resposta da BRS 137 quanto à irrigação (Tabela 2). Já a cultivar BRS 138 aumentou (13\%) o número de grãos por legume quando recebeu irrigação, igualando-se à BRS 137. O número de grãos por legume é uma característica pouco influenciada pela deficiência hídrica em cultivares de soja com hábito de crescimento determinado (Thomas \& Costa, 1994), pois os genótipos são selecionados para terem, na maioria, legumes com dois grãos. Sem irrigação, a cultivar BRS 137 apresentou $11 \%$ mais grãos por legume do que a BRS 138, e com irrigação, não houve diferença entre os genótipos. A análise dos efeitos simples indica que, na média dos tratamentos, a cultivar BRS 137 apresentou 5\% mais grãos por legume do que a BRS 138, e que a irrigação promoveu aumento de $7 \%$ nesse parâmetro.

As plantas que receberam irrigação produziram grãos mais pesados (Tabela 3). Em média, o peso de 100 grãos foi 3,3 g superior ao das plantas que não receberam irrigação. Tal diferença foi observa-

Tabela 2. Grãos por legume das cultivares de soja BRS 137 e BRS 138 em dois regimes hídricos. Eldorado do Sul, RS, 1998/1999(1).

\begin{tabular}{lccc}
\hline Cultivar & Não irrigado & Irrigado & Média \\
\hline BRS 137 & $2,20 \mathrm{Aa}$ & $2,25 \mathrm{Aa}$ & 2,22 \\
BRS 138 & $1,99 \mathrm{Bb}$ & $2,24 \mathrm{Aa}$ & 2,12 \\
\hline Média & 2,10 & 2,24 & 2,17 \\
\hline \multicolumn{4}{c}{ Médias seguidas pela mesma letra, maiúscula na linha e minúscula na } \\
coluna, não diferem entre si pelo teste unilateral de Dunnett a 5\% de pro- \\
babilidade; o coeficiente de variação foi de $1,2 \%$ e 2,5\% em relação a \\
irrigação e cultivar, respectivamente.
\end{tabular}

Pesq. agropec. bras., Brasília, v. 38, n. 2, p. 225-231, fev. 2003 
da em grãos provenientes de legumes contendo um, dois e três grãos. Quando ocorre deficiência hídrica durante o período de enchimento de grãos da soja, há redução no tamanho e peso do grão, decorrente da diminuição do suprimento de fotoassimilados pela planta ou da inibição do metabolismo do grão (Westgate et al., 1989; Salinas et al., 1996).

O menor peso de 100 grãos das plantas que sofreram deficiência hídrica também pode ser o resultado do período de enchimento de grãos mais curto do que o das plantas do tratamento irrigado. Falta de água e altas temperaturas aceleram o ciclo da soja a partir da metade final do período reprodutivo, enquanto a suplementação de água durante esse período faz com que o ciclo tenha a duração normal da cultivar (Korte et al., 1983a). A taxa de enchimento normalmente não influencia o peso dos grãos, pois ela varia pouco em virtude da disponibilidade hídrica para a planta de soja (Westgate et al., 1989), conseqüentemente, a duração do enchimento pode ter sido o fator determinante da diferença. Em média, a cultivar BRS 137 apresentou grãos 26\% mais pesados do que a BRS 138 (Tabela 3).

A soja apresenta potencial para ultrapassar o teto médio calculado na floração neste ano $\left(15.295 \mathrm{~kg} \mathrm{ha}^{-1}\right)$ bem como o relatado por Ventimiglia et al. (1999), que foi de $18.000 \mathrm{~kg} \mathrm{ha}^{-1}$. Não existem evidências de que o teto do potencial de rendimento tenha sido alcançado, nem que isto possa ocorrer, uma vez que o rendimento pode ser continuamente aumentado por meio do melhoramento de resistência a pragas, a doenças e a estresses ambientais e precisão no manejo da cultura, proporcionando melhor aproveitamento dos recursos disponíveis (Evans \& Fischer, 1999; Sprecht et al., 1999). Deve-se salientar a importância da identificação e utilização de genótipos

Tabela 3. Peso de 100 grãos de legumes com um, dois e três grãos das cultivares de soja BRS 137 e BRS 138 em dois regimes hídricos. Eldorado do Sul, RS, 1998/1999(1).

\begin{tabular}{lcccc}
\hline Legumes & BRS 137 & BRS 138 & Não irrigado & Irrigado \\
\hline Um grão & $17,3 \mathrm{a}$ & $13,6 \mathrm{~b}$ & $13,5 \mathrm{~b}$ & $17,5 \mathrm{a}$ \\
Dois grãos & $17,6 \mathrm{a}$ & $13,8 \mathrm{~b}$ & $14,0 \mathrm{~b}$ & $17,5 \mathrm{a}$ \\
Três grãos & $17,2 \mathrm{a}$ & $13,7 \mathrm{~b}$ & $14,0 \mathrm{~b}$ & $17,0 \mathrm{a}$ \\
\hline Média & $17,3 \mathrm{a}$ & $13,7 \mathrm{~b}$ & $13,8 \mathrm{~b}$ & $17,1 \mathrm{a}$ \\
\hline
\end{tabular}

(1)Médias seguidas da mesma letra, na linha, não diferem entre si pelo teste unilateral de Dunnett a 5\% de probabilidade. com elevado potencial de rendimento quando se emprega nível alto de tecnologia na lavoura, uma vez que esse maior potencial, aliado a práticas de manejo aplicadas em estádios críticos, pode garantir rendimentos mais elevados.

Os resultados obtidos no presente trabalho podem ser empregados na comparação do potencial de rendimento de lavouras durante a ontogenia, permitindo a tomada de decisões de manejo e direcionamento de recursos que privilegiem lavouras com maior potencial. Podem servir também como subsídios para a pesquisa, na busca de genótipos ou conjunto de práticas de manejo que maximizem o potencial de rendimento, além de sua possível utilização no desenvolvimento de modelos matemáticos capazes de estimar o potencial de rendimento o mais cedo possível durante a ontogenia da soja.

\section{Conclusões}

1. A irrigação proporciona incremento no potencial de rendimento de grãos, pela redução do aborto de flores e abscisão de legumes, aumento no peso dos grãos e número de grãos por legume.

2. A modificação do espaçamento entre linhas não afeta o potencial de rendimento de grãos durante a ontogenia das duas cultivares testadas.

3. A cultivar BRS 137 tem potencial de rendimento de grãos mais elevado do que a BRS 138, decorrente da grande produção de flores, maior número de grãos por legume e grãos mais pesados.

\section{Referências}

BARTZ, H. R. (Coord.). Recomendações de adubação e calagem para os Estados do Rio Grande do Sul e de Santa Catarina. Passo Fundo: Sociedade Brasileira de Ciência do Solo, 1994. 224 p.

BERGAMASCHI, H.; GUADAGNIN, M. R. Agroclima da Estação Experimental Agronômica/UFRGS. Porto Alegre: UFRGS, 1990. 60 p.

BOARD, J. E.; KAMAL, M.; HARVILLE, B. G. Temporal importance of greater light interception to increased yield in narrow-row soybean. Agronomy Journal, Madison, v. 84, n. 4, p. 575-579, 1992.

BOERMA, H. R.; ASHLEY, D. A. Irrigation, row spacing, and genotype effects on late and ultralate planted soybeans. 
Agronomy Journal, Madison, v. 74, n. 6, p. 995-999, 1982.

COSTA, J. A.; MARCHEZAN, E. Características dos estádios de desenvolvimento da soja. Campinas: Fundação Cargill, 1982.30 p.

DOMINGUEZ, C.; HUME, D. J. Flowering, abortion and yield of early maturing soybean at three densities. Agronomy Journal, Madison, v. 70, n. 5, p. 801-805, 1978.

EMBRAPA. Centro Nacional de Pesquisa de Solos (Rio de Janeiro, RJ). Sistema brasileiro de classificação de solos. Brasília: Embrapa-SPI/Embrapa-CNPS, 1999. $412 \mathrm{p}$.

EVANS, L. T.; FISCHER, R. A. Yield potential: its definition, measurement, and significance. Crop Science, Madison, v. 39, n. 6, p. 1544-1551, 1999.

HANSEN, W. R.; SHIBLES, R. Seasonal log of the flowering and podding activity of field-grown soybeans. Agronomy Journal, Madison, v. 70, n. 1, p. 47-50, 1978.

KORTE, L. L.; SPECHT, J. E.; WILLIAMS, J. H.; SORENSEN, R. C. Irrigation of soybean genotypes during reproductive ontogeny II: yield component responses. Crop Science, Madison, v. 23, n. 3, p. 528-533, 1983 a.

KORTE, L. L.; WILLIAMS, J. H.; SPECHT, J. E.; SORENSEN, R. C. Irrigation of soybean genotypes during reproductive ontogeny I: agronomic responses. Crop Science, Madison, v. 23, n. 3, p. 521-527, 1983b.

LOOMIS, R. S.; CONNOR, D. J. Crop ecology: productivity and management in agricultural systems. Cambridge, Inglaterra: Cambridge University Press, 1992. $520 \mathrm{p}$.

MARCHEZAN, E.; COSTA, J. A. Produção e fixação de flores e legumes, em três cultivares de soja. Pesquisa Agropecuária Brasileira, Brasília, v. 18, n. 2, p. 129-136, fev. 1983.

NAVARRO JUNIOR, H. M. Estratégias associadas à expressão do potencial de produção por planta em cultivares de soja. 1998. 82 f. Dissertação (Mestrado em Fitotecnia) - Universidade Federal do Rio Grande do Sul, Porto Alegre, 1998.

PIRES, J. L. F.; COSTA, J. A.; THOMAS, A. L.; MAEHLER, A. R. Efeito de populações e espaçamentos sobre o potencial de rendimento da soja durante a ontogenia. Pesquisa Agropecuária Brasileira, Brasília, v. 35 , n. 8 , p. 1541-1547, ago. 2000.

QUEIROZ, E. F.; MOLION, L. C. B.; MINOR, H. C.; NEUMAIER, N. Um modelo matemático de quantificação do efeito da disponibilidade hídrica em soja. Pesquisa Agropecuária Brasileira, Brasília, v. 31, n. 10, p. 683690, out. 1996.

ROLIM, G. S.; SENTELHAS, P. C.; BARBIERI, V. Planilhas no ambiente EXCEL ${ }^{\mathrm{TM}}$ para os cálculos de balanços hídricos: normal, seqüencial, de cultura e de produtividade real e potencial. Revista Brasileira deAgrometeorologia, Santa Maria, v. 6, n. 1, p. 133-137, 1998.

SALINAS, A. R.; ZELENER, N.; CRAVIOTTO, R. M.; BISARO, V. Respuestas fisiológicas que caracterizan el comportamiento de diferentes cultivares de soja a la deficiencia hídrica. Pesquisa Agropecuária Brasileira, Brasília, v. 31, n. 5, p. 331-338, maio 1996.

SCHAIK, P. H. van; PROBST, A. H. The inheritance of inflorescence type peduncle length, flowers per node, and percent flower shedding in soybeans. Agronomy Journal, Madison, v. 50, n. 2, p. 98-102, 1958.

SIONIT, N.; KRAMER, P. J. Effects of water stress during different stages of growth of soybean. Agronomy Journal, Madison, v. 69, n. 2, p. 274-278, 1977.

SPRECHT, J. E.; HUME, D. J.; KUMUDINI, S. V. Soybean yield potential: a genetic and physiological perspective. Crop Science, Madison, v. 39, n. 6, p. 1560 1570, 1999.

THOMAS, A. L.; COSTA, J. A. Influência do déficit hídrico sobre o desenvolvimento e rendimento da soja. Pesquisa Agropecuária Brasileira, Brasília, v. 29, n. 9, p. 1389-1396, set. 1994.

VENTIMIGLIA, L. A.; COSTA, J. A.; THOMAS, A. L.; PIRES, J. L. F. Potencial de rendimento da soja em razão da disponibilidade de fósforo no solo e dos espaçamentos. Pesquisa Agropecuária Brasileira, Brasília, v. 34, n. 2, p. 195-199, fev. 1999.

WESTGATE, M. E.; SCHUSSLER, J. R.; REICOSKY, D. C. Effect of water deficits on seed development in soybean. Plant Physiology, Lancaster, v. 91, n. 2, p. 980985, 1989. 